\title{
COMMUTATION PROPERTIES OF OPERATOR POLYNOMIALS
}

\author{
S. R. CARADUS \\ (Received 5 May 1969; revised 8 August 1969) \\ Communicated by E. Strzelecki
}

Suppose $A$ and $B$ are continuous linear operators mapping a complex Banach space $X$ into itself. For any polynomial $p$ over $C$, it is obvious that when $A$ commutes with $B$, then $p(A)$ commutes with $B$. To see that the reverse implication is false, let $A$ be nilpotent of order $n$. Then $A^{n}$ commutes with all $B$ but $A$ cannot do so. Sufficient conditions for the implication: $p(A)$ commutes with $B$ implies $A$ commutes with $B$ : were given by Embry [2] for the case $p(\lambda)=\lambda^{n}$ and Finkelstein and Lebow [3] in the general case. The latter authors proved in fact that if $f$ is a function holomorphic on $\sigma(A)$ and if $f$ is univalent with non-vanishing derivative on $\sigma(A)$, then $A$ can be expressed as a function of $f(A)$.

In this paper, similar questions are studied when $A$ and $B$ are closed operators with domain and range in $X$. Immediately the question of the definition of commutativity arises. Several definitions appear in the literature. A well-known approach is

$C_{1}: B$ commutes with $A$ iff $D(B)$, the domain of $B$ is all of $X$ and $A B$ is an extension of $B A$.

See, for example, [5].

More recently, Marti [4] used the condition:

$C_{2}: B$ commutes with $A$ iff $D(A) \subseteq D(B), B D(A) \subseteq D(A)$ and

for all $x \in D(B A)$.

$$
A B x=B A x
$$

It is a simple exercise to show that $C_{1}$ implies $C_{2}$. Both $C_{1}$ and $C_{2}$ suffer from an evident lack of symmetry. A symmetrical definition appears in [1]:

$C_{3}: B$ commutes with $A$ iff $D(A) \cap D(A B)=D(B) \cap D(B A)$ and

$$
A B x=B A x
$$

for all $x \in D(A B) \cap D(B A)$.

Again, it is straightforward to verify that $C_{2}$ implies $C_{3}$. Moreover, if $D(B)=X$, then $C_{3}$ implies $C_{1}$. If $A$ and $B$ are closed operators with non empty resolvent sets, 
then from [1], we know that $C_{3}$ is a necessary and sufficient condition for the commutativity of the resolvent operators.

In that which follows, we obtain a sufficient condition that the $C_{3}$-commutativity of $p(A)$ with $B$ should imply the $C_{3}$-commutativity of $A$ with $B$ when $A$ and $B$ are closed operators with non empty resolvent sets. Suppose that $p$ is a monic polynomial of degree $n$ and let $\lambda_{0} \in \rho(A)$. If $\mu_{1}, \mu_{2}, \cdots, \mu_{n}$ denote the roots of $p(\mu)=p\left(\lambda_{0}\right)$ with $\mu_{1}=\lambda_{0}$ then, since $\rho(A)$ is an open set we can assume without loss of generality that $p^{\prime}\left(\mu_{k}\right) \neq 0$ for $k=1,2, \cdots, n$ and that the $\mu_{k}$ are distinct. In these terms we can state

THEOREM. Suppose that $p(A)$ commutes with $B$ in the $C_{3}$ sense. Suppose also that for some $\lambda_{0} \in \rho(A)$ we have

$$
\sum_{k=1}^{n} \frac{1}{p^{\prime}\left(\mu_{k}\right)\left(\lambda_{1}-\mu_{k}\right)\left(\lambda_{2}-\mu_{k}\right)} \neq 0
$$

for all $\lambda_{1}, \lambda_{2} \in \sigma(A)$. Then $A$ commutes with $B$ in the $C_{3}$ sense.

Proof. Since $p(\mu)-p\left(\lambda_{0}\right)=\prod_{k=1}^{n}\left(\mu-\mu_{k}\right)$ and the $\mu_{k}$ are distinct, we can write $\left[p(\mu)-p\left(\lambda_{0}\right)\right]^{-1}=\sum_{k=1}^{n} a_{k}\left(\mu-\mu_{k}\right)^{-1}$ and hence

Moreover

$$
a_{k}=\lim _{\mu \rightarrow \mu_{k}} \frac{\mu-\mu_{k}}{p(\mu)-p\left(\lambda_{0}\right)}=\lim _{\mu \rightarrow \mu_{k}} \frac{\mu-\mu_{k}}{p(\mu)-p\left(\mu_{k}\right)}=\frac{1}{p^{\prime}\left(\mu_{k}\right)} .
$$

so that

$$
\begin{aligned}
\left(A-\mu_{k}\right)^{-1} & =\left[\left(A-\mu_{1}\right)\left[I+\left(A-\mu_{1}\right)^{-1}\left(\mu_{1}-\mu_{k}\right)\right]\right]^{-1} \\
& =\left[I+\left(A-\lambda_{0}\right)^{-1}\left(\mu_{1}-\mu_{k}\right)\right]^{-1}\left(A-\lambda_{0}\right)^{-1},
\end{aligned}
$$

If we define

$$
\left[p(A)-p\left(\lambda_{0}\right)\right]^{-1}=\sum_{k=1}^{n} \frac{\left[I+\left(A-\lambda_{0}\right)^{-1}\left(\mu_{1}-\mu_{k}\right)\right]^{-1}\left(A-\lambda_{0}\right)^{-1}}{p^{\prime}\left(\mu_{k}\right)} .
$$

$$
f(\lambda)=\sum_{k=1}^{n} \frac{\lambda}{p^{\prime}\left(\mu_{k}\right)\left[1-\left(\mu_{1}-\mu_{k}\right) \lambda\right]}
$$

then $\left[p(A)-p\left(\lambda_{0}\right)\right]^{-1}=f\left[\left(A-\lambda_{0}\right)^{-1}\right]$. If $f$ fulfils the requirements of the result of Finkelstein and Lebow, then we can conclude that $\left(A-\lambda_{0}\right)^{-1}$ is a function of $\left[p(A)-p\left(\lambda_{0}\right)\right]^{-1}$ and hence the result follows.

Consider now the properties of $f$. Evidently $f$ is analytic except when $\lambda=$ $\left(\mu_{k}-\lambda_{0}\right)^{-1}$. Now since $p(A)-p\left(\lambda_{0}\right)=\prod_{k=1}^{n}\left(A-\mu_{k}\right)$ it is evident that all $\mu_{k}$ belong to $\rho(A)$. Hence $\left(\mu_{k}-\lambda_{0}\right)^{-1} \in \rho\left[\left(A-\lambda_{0}\right)^{-1}\right]$ so that $f$ is analytic on $\sigma\left[\left(A-\lambda_{0}\right)^{-1}\right]$. It remains to show that the restriction of $f$ to $\sigma\left[\left(A-\lambda_{0}\right)^{-1}\right]$ is univalent with nonvanishing derivative. Straightforward calculations show that this requirement is precisely the assumed property (1). For example, if $\theta_{1}, \theta_{2} \in \sigma\left[\left(A-\lambda_{0}\right)^{-1}\right]$ then there exists $\lambda_{1}, \lambda_{2} \in \sigma(A)$ such that $\left(\lambda_{i}-\lambda_{0}\right)^{-1}=\theta_{i}, i=1,2$. Suppose $\theta_{1} \neq \theta_{2}$ but $f\left(\theta_{1}\right)=f\left(\theta_{2}\right)$; then 


$$
\sum_{k=1}^{n}\left\{\left(\lambda_{1}-\lambda_{0}\right) p^{\prime}\left(\mu_{k}\right)_{i}\left[1+\frac{\mu_{1}-\mu_{k}}{\lambda_{1}-\lambda_{0}}\right]\right\}^{-1}=\sum_{k=1}^{n}\left\{\left(\lambda_{2}-\lambda_{0}\right) p^{\prime}\left(\mu_{k}\right)\left[1+\frac{\mu_{1}-\mu_{k}}{\lambda_{1}-\lambda_{0}}\right]\right\}^{-1}
$$

which reduces to

$$
\begin{aligned}
& \sum_{k=1}^{n}\left[p^{\prime}\left(\mu_{k}\right)\left(\lambda_{1}-\mu_{k}\right)\right]^{-1}=\sum_{k=1}^{n}\left[p^{\prime}\left(\mu_{k}\right)\left(\lambda_{2}-\mu_{k}\right)\right]^{-1} \text { i.e. } \\
& \sum_{k=1}^{n}\left[p^{\prime}\left(\mu_{k}\right)\left(\lambda_{1}-\mu_{k}\right)\left(\lambda_{2}-\mu_{k}\right)\right]^{-1}=0 .
\end{aligned}
$$

Since this contradicts (1), we know that $f$ is univalent on $\sigma\left[\left(A-\lambda_{0}\right)^{-1}\right]$. In a similar way, the assumption that $f^{\prime}\left(\theta_{1}\right)=0$ leads to equation (1) with $\lambda_{1}=\lambda_{2}$. This concludes the proof.

REMARK. The relation of the result of our theorem and the results of [2] and [3] seems obscure. Even when $A$ and $B$ are in $B(X)$ and $p(\lambda)=\lambda^{n},(1)$ reduces to

$$
\sum_{k=1}^{n} \frac{\omega^{k}}{\left(\omega^{k} \lambda_{1}-\lambda_{0}\right)\left(\omega^{k} \lambda_{2}-\lambda_{0}\right)} \neq 0 \quad \text { for } \lambda_{1}, \lambda_{2} \in \sigma(A)
$$

where $\omega=\exp (2 i \pi / n)$. It is not obvious that this condition is related in any simple way to that of [2]: $\sigma(A) \cap \sigma\left(\omega^{k} A\right)=\phi, k=2,3, \cdots, n$. However when $n=2$, (3) reduces to $\lambda_{0}\left(\lambda_{1}+\lambda_{2}\right) \neq 0$ so that (1) is equivalent to the condition of [2].

Corollary. If $\sigma(A)=\phi$ and $p(A)$ commutes with $B$ in the $C_{3}$ sense, then $A$ commutes with $B$ in the $C_{3}$ sense.

\section{References}

[1] S. R. Caradus, 'A Note on a Paper by J. T. Marti', Comment. Math. Helv. 44 (3) (1969), $282-283$.

[2] M. R. Embry, 'Nth Roots of Operators', Proc. Amer. Math. Soc. 19 (1) (1968), 63-68.

[3] M. Finkelstein and A. Lebow, 'A Note on Nth Roots of Operators', Proc. Amer. Math. Soc. $21(1969), 250$.

[4] J. T. Marti, 'Operational Calculus for Two Commuting Closed Operators', Comment. Math. Helv. 43 (1968), 87-97.

[5] M. H. Stone, 'On Unbounded Operators in Hilbert Space', J. Indian Math. Soc. (N.S.) 15 (1951), 155-192.

Australian National University, Canberra

Queen's University at Kingston 